ORIGINAL ARTICLE

\title{
Involvement of polypyrimidine tract-binding protein (PTBP1) in maintaining breast cancer cell growth and malignant properties
}

\author{
X He ${ }^{1,2}$, AD Arslan ${ }^{3,5}$, T-T Ho ${ }^{3,6}$, C Yuan ${ }^{1}$, MR Stampfer ${ }^{4}$ and WT Beck ${ }^{2,3}$
}

\begin{abstract}
We have investigated some roles of splicing factor polypyrimidine tract-binding protein (PTBP1) in human breast cancer. We found that PTBP1 was upregulated in progressively transformed human mammary epithelial cells (HMECs), as well as in breast tumor cell lines compared with HMECs with finite growth potential and found that the level of PTBP1 correlated with the transformation state of HMECs. Knockdown of PTBP1 expression substantially inhibited tumor cell growth, colony formation in soft agar and in vitro invasiveness of breast cancer cell lines, a result similar to what we have reported in ovarian cancer. However, ectopic expression of PTBP1 (as a PTBP1-EGFP fusion protein) did not enhance the proliferation of immortalized HMEC. Rather, PTBP1 expression promoted anchorage-independent growth of an immortalized HMEC as assessed by increased colony formation in soft agar. In addition, we found that knockdown of PTBP1 expression led to upregulation of the expression of the M1 isoform of pyruvate kinase (PKM1) and increase of the ratio of PKM1 vs PKM2. PKM1 has been reported to promote oxidative phosphorylation and reduce tumorigenesis. Correspondingly, we observed increased oxygen consumption in PTBP1-knockdown breast cancer cells. Together, these results suggest that PTBP1 is associated with breast tumorigenesis and appears to be required for tumor cell growth and maintenance of transformed properties. PTBP1 exerts these effects, in part, by regulating the splicing of pyruvate kinase, and consequently alters glucose metabolism and contributes to the Warburg effect.
\end{abstract}

Oncogenesis (2014) 3, e84; doi:10.1038/oncsis.2013.47; published online 13 January 2014

Subject Categories: Cellular oncogenes

Keywords: breast cancer; polypyrimidine tract-binding protein; RNA interference; human mammary epithelial cell; tumorigenesis

\section{INTRODUCTION}

Breast cancer is the most common malignant disease in US women and is a leading cause of cancer mortality. ${ }^{1}$ Despite years of intensive study and substantial progress, our understanding of the mechanisms that mediate the development and progression of breast cancer is still incomplete. Many factors that function as oncogenes or tumor suppressor genes in breast cancer have been identified, ${ }^{2-5}$ in addition to well-known breast cancer-associated genes such as $B R C A 1, B R C A 2, p 53$, estrogen receptor and the epidermal growth factor receptor family, ${ }^{6}$ and microRNAs also affect the regulation of breast cancer cell growth and metastasis. ${ }^{7,8}$ We present data herein showing that a splicing factor, polypyrimidine tract-binding protein (PTBP1), is important in maintaining breast cancer cell growth and malignant properties, and suggest that it can be added to this growing list of genes associated with breast cancer.

PTBP1 is an RNA-binding protein with various molecular functions related to RNA metabolism. It is a major repressive regulator of alternative splicing, causing exon skipping in numerous alternatively spliced pre-mRNAs. ${ }^{9}$ It is involved in the $3^{\prime}$-end processing of mRNA, affecting $3^{\prime}$-end cleavage and polyadenylation. ${ }^{10,11}$ PTBP1 was also found to have a role in the control of mRNA stability: binding of PTBP1 to the $3^{\prime}$-untranslated region has been shown to increase the stability of mRNAs such as rat insulin, vascular endothelial growth factor and CD154. ${ }^{12-15}$ PTBP1 also has a role in determining mRNA localization in the cytoplasm. $^{16}$ Another important function of PTBP1 is its involvement in the internal ribosome entry site-mediated translation. ${ }^{17}$ Indeed, it has been suggested that PTBP1 may act as an RNA chaperone to help internal ribosome entry site attain correct conformation to permit translation initiation. ${ }^{18,19}$

PTBP1 is ubiquitously expressed but its levels among different tissues and cells vary substantially. ${ }^{20}$ We and others have found that PTBP1 is overexpressed in human epithelial ovarian tumors $^{21,22}$ and glioblastomas ${ }^{23}$ compared with normal tissues. Knockdown of PTBP1 expression in tumor cell lines, such as A2780 (ovarian cancer) and PC-3M (prostate cancer), significantly impaired tumor cell growth and their malignant properties. ${ }^{22,24}$ However, PTBP1 knockdown had dual effects on HeLa cells: proliferation and anchorage-independent growth (AIG) were reduced but the invasive behavior of the cell line was enhanced. ${ }^{24}$

In the present study, we investigated the role of PTBP1 in breast cancer. We found that PTBP1 was overexpressed in immortalized human mammary epithelial cells (HMECs) and breast cancer cell lines compared with finite HMEC strains. Suppression of PTBP1 expression by RNA interference substantially inhibited breast

${ }^{1}$ Department of Biopharmaceutical Sciences, College of Pharmacy-Rockford, University of Illinois at Chicago, Rockford, IL, USA; ${ }^{2}$ Cancer Center, University of Illinois, Chicago, IL, USA; ${ }^{3}$ Department of Biopharmaceutical Sciences, College of Pharmacy, University of Illinois at Chicago, Chicago, IL, USA and ${ }^{4}$ Lawrence Berkeley National Laboratory, Berkeley, CA, USA. Correspondence: Dr X He, Department of Biopharmaceutical Sciences, College of Pharmacy-Rockford, University of Illinois at Chicago, 1601 Parkview Avenue, Room N308, Rockford, IL 61107, USA or Dr WT Beck, Department of Biopharmaceutical Sciences, College of Pharmacy, University of Illinois at Chicago, 833 South Wood Street, 335 Pharm, MC 865, Chicago, IL 60612, USA.

E-mail: xiaohe@uic.edu or WTBeck@uic.edu

${ }^{5}$ Current address: Robert H Lurie Comprehensive Cancer Center, Northwestern University, Chicago, IL, USA.

${ }^{6}$ Current address: Cancer Institute, University of Mississippi Medical Center, Jackson, MS, USA.

Received 20 August 2013; revised 13 November 2013; accepted 14 November 2013 
cancer cell growth and malignant behavior, which was accompanied by increased ratio of M1 (PKM1) vs M2 isoforms of pyruvate kinase (PKM2) and oxygen consumption. Ectopic expression of a PTBP1-EGFP (enhanced green fluorescent protein) fusion protein enhanced AIG of immortalized HMECs but not their anchorage-dependent growth. Together, these results suggest that PTBP1 is involved in breast tumorigenesis.

\section{RESULTS}

PTBP1 expression is upregulated in immortalized HMECs and breast cancer cell lines

We examined by western blotting the expression of PTBP1 in a series of progressively transformed HMECs as well as in the breast cancer cell lines MCF-7, T47D and MDA-MB231. The panel of HMECs includes finite lifespan post-stasis HMEC (HMEC 184), carcinogen-exposed, extended-life cultures with finite lifespan (HMEC 184Aa, derived from specimen 184), ${ }^{25}$ immortalized HMEC with indefinite lifespan but no capability for AIG (HMEC 184A1, derived from 184Aa) ${ }^{25}$ and immortalized HMEC with indefinite lifespan and AIG (HMEC 184AA2 and HMEC 184AA3, derived from $184 \mathrm{Aa}){ }^{26}$ As shown in Figure 1 , the expression of PTBP1 is upregulated in immortalized HMECs (184A1, 184AA2 and 184AA3) as well as in breast cancer cell lines, compared with HMECs with finite lifespan (184 and 184Aa). Moreover, the level of PTBP1 is considerably higher in malignant transformed HMECs (184AA2 and 184AA3) and breast cancer cells than in HMEC without malignant properties (184A1). These results indicate that PTBP1 is associated with, and may be involved in, the neoplastic transformation of HMEC; its upregulation is likely an early event in the transformation process.

Ectopic expression of PTBP1 promotes AIG of immortalized HMEC To determine whether PTBP1 has a role in the transformation of HMEC, we used lentiviruses to ectopically express the PTBP1-EGFP fusion protein in HMEC 184A1 cells. We chose $184 \mathrm{~A} 1$ cells to express PTBP1-EGFP because these cells can propagate indefinitely but lack other properties of malignant transformation. The endogenous PTBP1 levels in 184A1 are upregulated compared with its finite precursor, 184Aa, but are lower than that in other immortalized HMEC and breast cancer cell lines (see Figure 1). After confirmation of ectopic expression of the PTBP1-EGFP fusion protein in 184A1, as shown in Figure 2a, we compared the growth of 184A1 cells expressing PTBP1-EGFP (184A1/PTBP1-EGFP) with $184 \mathrm{~A} 1$ cells carrying the control vector (expressing EGFP only) (184A1/LV), parental 184A1 cells and 184AA2 cells. We found no significant difference in their growth curves (Figure 2b). However, we observed changes in morphology of PTBP1-overexpressing cells: 184A1/PTBP1-EGFP cells grew in tight patches in a manner similar to 184AA2, but different than 184A1/LV and parental $184 \mathrm{~A} 1$ cells (Figure $2 \mathrm{C}$ ). We then examined the AIG of these various cell lines and found that 184A1/PTBP1-EGFP, 184AA2 and MCF-7 formed 4.8-, 3.3- and 9.4-fold, respectively, as many colonies as 184A1/LV (Figure 2d). These results indicate that PTBP1-EGFP promotes AIG of 184A1 cells.

Knockdown of PTBP1 expression inhibits breast cancer cell growth To address the question whether the overexpressed PTBP1 has any functional role in breast cancer cells, we established sublines of breast cancer cell lines MCF-7, T47D and MDA-MB231 to express doxycycline (DOXY)-induced PTBP1 small interfering RNAs (siRNAs) or a control luciferase siRNA. As detailed in the Materials and Methods, we generated these sublines by sequential transduction of parental cell lines first with lentiviruses carrying the fusion protein $\mathrm{TTR}-\mathrm{KRAB}$, which binds to the tetO element in the absence of DOXY and suppresses the transcription of nearby genes within a distance of up to $3 \mathrm{~kb}$ from its binding site, ${ }^{27}$ and then with lentiviruses carrying expression cassettes of short hairpin RNAs (shRNAs). We tested two effective PTBP1 siRNAs that target different regions of the PTBP1 mRNA, PTBP1si1 and PTBP1si3, ${ }^{22}$ in this study, and we isolated the corresponding sublines called MCF-7/PTBP1si1, MCF-7/PTBP1si3, MDA-MB231/ PTBP1si1, MDA-MB231/PTBP1si3, T47D/PTBP1si1 and T47D/ PTBP1si3. The control siRNA targets the transcript of firefly luciferase ${ }^{28}$ and the corresponding sublines were called MCF-7/ LUCsi, MDA-MB231/LUCsi and T47D/LUCsi. As shown in Figure 3, PTBP1 expression is substantially suppressed in PTBP1si1 and PTBP1si3 sublines grown with DOXY but not in control sublines.

We then examined the growth of the sublines in the presence or absence of DOXY. As shown in Figure 4, the growth of PTBP1si1 and PTBP1si3 sublines was substantially inhibited when grown in the presence of DOXY, that is, with PTBP1 knocked down, while the growth of control sublines showed no significant difference between DOXY and no DOXY treatment. For MCF-7 and MDAMB231 sublines, we tested two or more clones and obtained similar results. For T47D sublines, we did not isolate individual clones. Instead, we pooled lentivirus-infected cells to examine their growth.

Knockdown of PTBP1 expression inhibits AIG of breast cancer cells To determine whether overexpressed PTBP1 in breast cancer cells is required for AIG, a feature that is common in cancer cells, we performed colony formation assays in soft agar with MCF-7 and T47D sublines. As shown in Figure 5, MCF-7/PTBP1si1, MCF-7/ PTBP1si3, T47D/PTBP1si1 and T47D/PTBP1si3 formed substantially fewer colonies (10-20\%) when they were grown with DOXY (PTBP1 knocked down) than without DOXY, indicating that PTBP1 knockdown suppressed MCF-7 and T47D cells' capability for AIG.
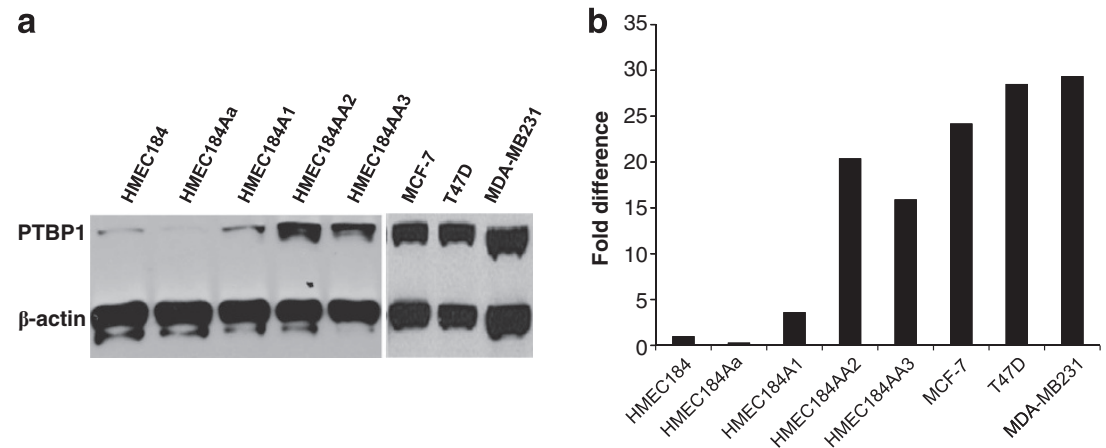

Figure 1. Overexpression of PTBP1 in progressively transformed HMECs and breast cancer cell lines. (a) Western blot. See the Results for the description of HMECs. (b) Fold differences of PTBP1 expression between HMEC184 and other cells after normalization to $\beta$-actin. 
a

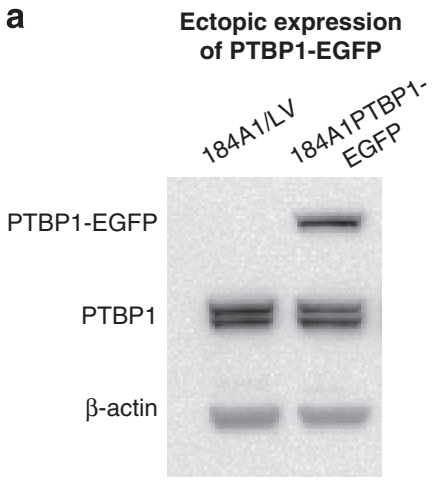

b

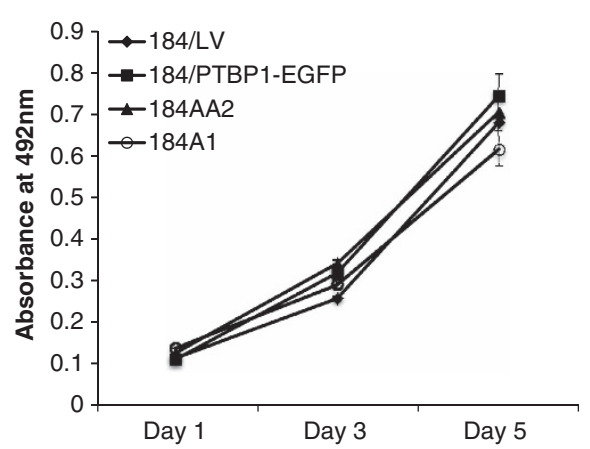

c 184A1
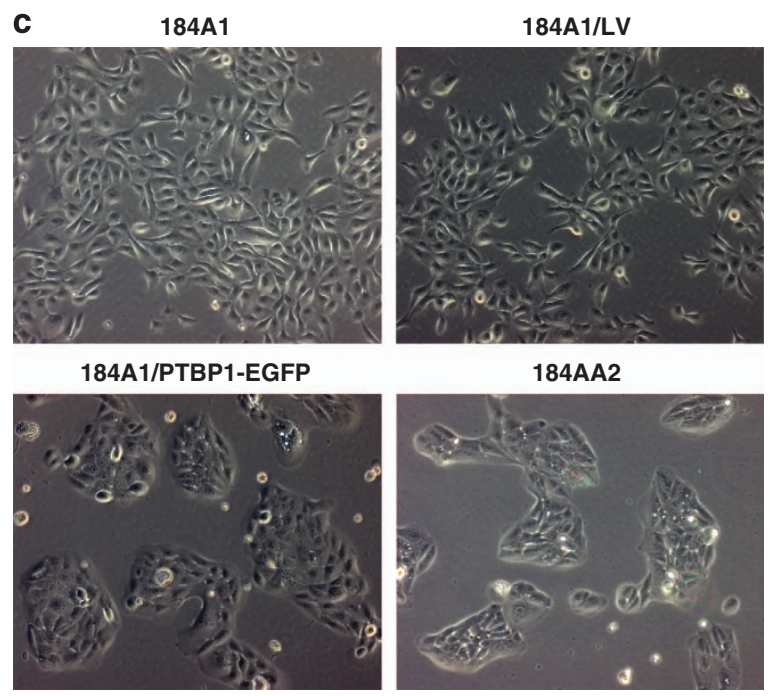

184A1/PTBP1-EGFP

d Colony formation in soft agar

184A1/LV 184A1/ PTBP1-EGFP

184AA2 MCF-7
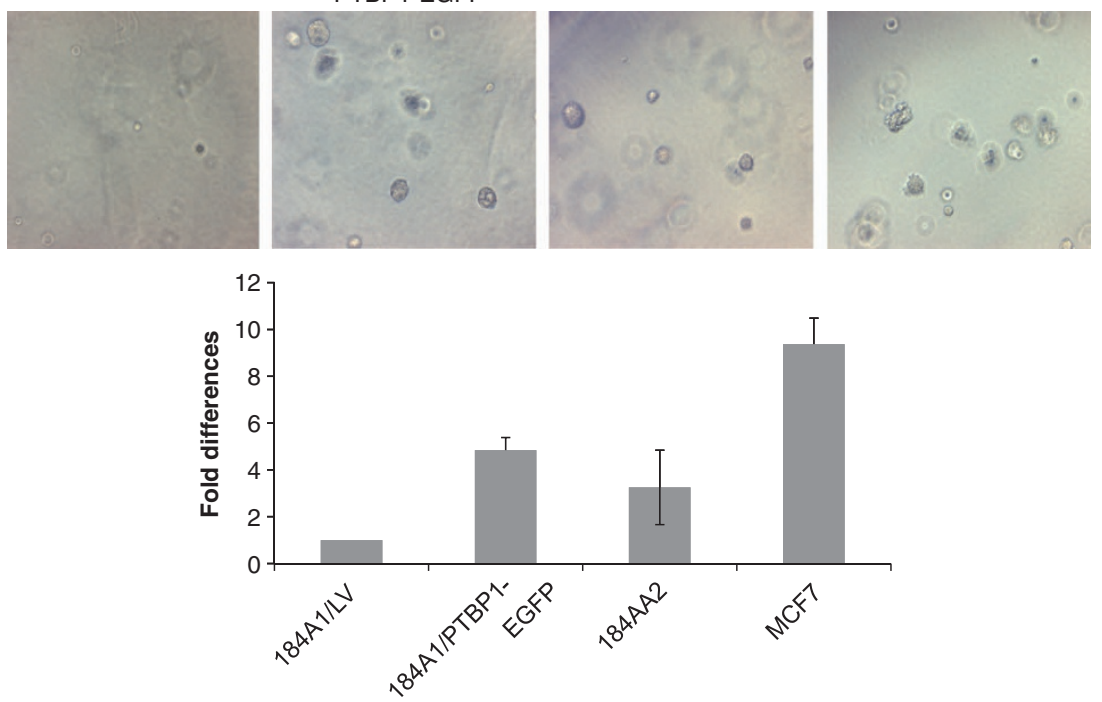

Figure 2. Ectopic expression of PTBP1 promotes AIG of HMEC 184A1 cells. (a) Western blot showing the expression of PTBP1-EGFP fusion protein in 184A1 cells. (b) Cell growth curves determined by MTT assay. (c) Micrographs showing the appearance of parental 184A1 cells, 184A1 cells carrying control vector (184A1/LV), 184A1 cells expressing PTBP1-EGFP (184A1/PTBP1-EGFP) as well as 184AA2 cells. Magnification, $\times 100$. (d) Colony formation assay. Upper panel: sample micrographs showing the colonies formed in the agar matrix. Magnification, $\times 40$. Lower panel: fold differences of colony formation of 184A1/PTBP1-EGFP, 184AA2 and MCF-7 cells relative to the control cells $184 \mathrm{~A} 1 / \mathrm{LV}$. 


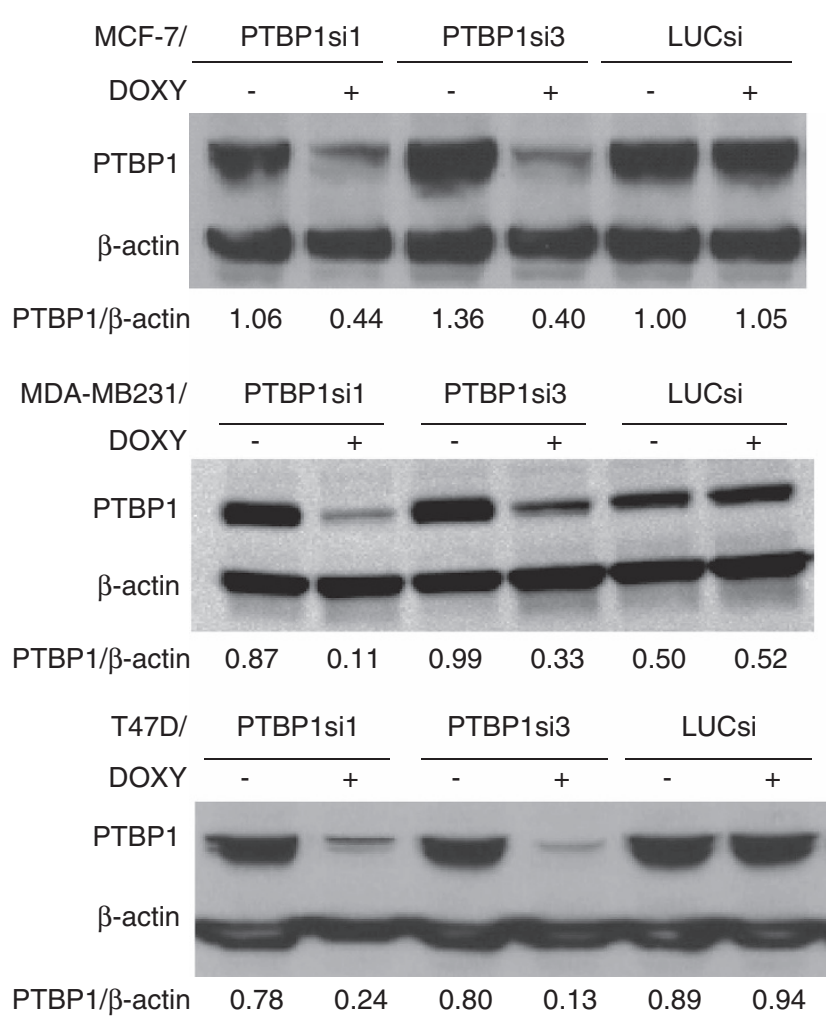

Figure 3. DOXY-inducible knockdown of PTBP1 expression in breast cancer cell lines. Shown are the results of western blotting for PTBP1 in subline cells derived from MCF-7, MDA-MB231 and T47D cell lines.
Knockdown of PTBP1 expression inhibits in vitro invasiveness of breast cancer cells

One hallmark of cancer cells is their invasive properties. ${ }^{29}$ To determine whether PTBP1 overexpression contributes to this malignant phenotype, we examined whether PTBP1 knockdown interfered with the in vitro invasiveness of MDA-MB231, a highly invasive 'triple-negative' breast cancer cell line. ${ }^{30}$ As shown in Figure 6, fewer MDA-MB231/PTBP1si1 and MDA-MB231/PTBP1si3 cells invaded Matrigel in the presence of DOXY (PTBP1 knocked down) than in the absence of DOXY, while the control subline MDA-MB231/LUCsi displayed similar invasive activity in both conditions. These results indicate that knockdown of PTBP1 indeed inhibits the invasive behavior of these triple-negative breast cancer cells, a finding that would appear to have therapeutic consequences for such basal-like breast cancer cells.

Knockdown of PTBP1 leads to upregulation of PKM1 and increased oxygen consumption

The Warburg effect or aerobic glycolysis is a characteristic of cancer cells. ${ }^{31}$ Recent studies found that this phenotype could be accounted for by an expression switch of PKM1 to the PKM2 isoform (PKM2), ${ }^{32}$ which also promotes tumorigenesis. PKM1 and PKM2 are encoded by the same gene and result from alternative splicing of mutually exclusive exons. ${ }^{32}$ PTBP1, along with heterogenous nuclear ribonucleoprotein $A 1$ and $A 2$, regulates this alternative splicing by enhancing PKM2 expression while repressing PKM1 expression. ${ }^{33,34}$ To determine whether there is a change in the expression of PKM1 and PKM2 in PTBP1-knockdown cells, we examined these two isoforms in MCF-7 and T47D sublines by reverse transcriptase-PCR and real-time quantitative PCR. As shown in Figure 7a, the ratio of PKM1 vs PKM2 increases

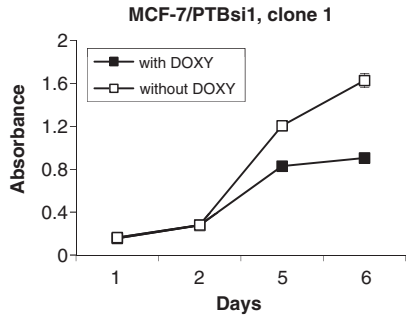

MDA-MB231/PTBP1si1, clone1
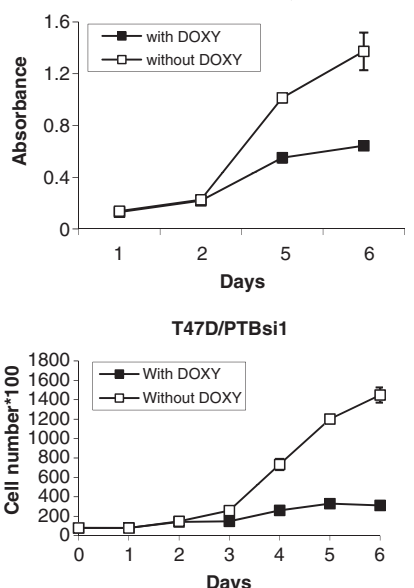

MCF-7/PTBsi3, clone 1

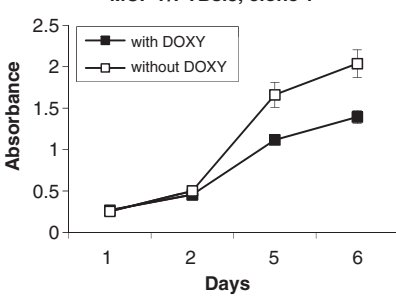

MDA-MB231/PTBP1si3, clone 1
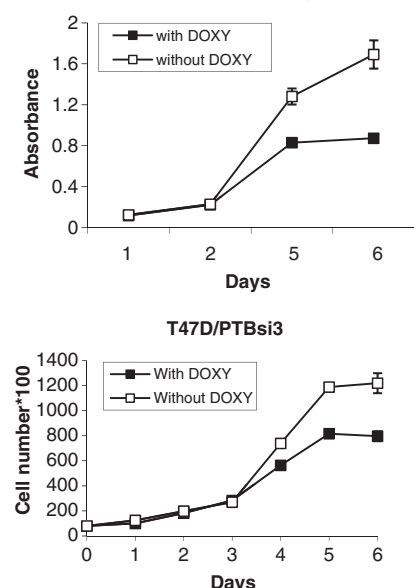

MCF-7/LUCsi, clone 1

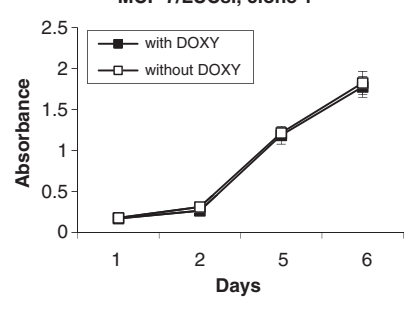

MDA-MB231/LUCsi, clone 1
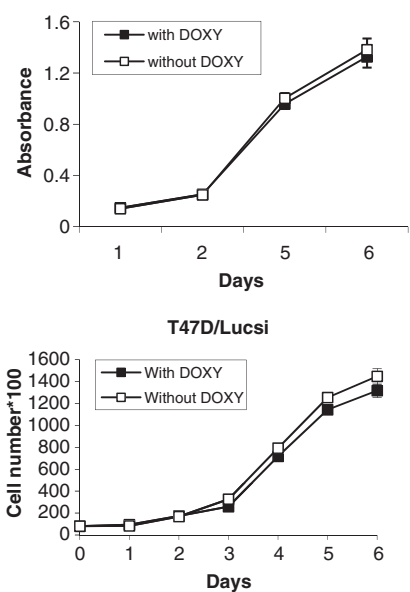

Figure 4. Knockdown of PTBP1 expression inhibits breast cancer cell growth. Cell growth curves of subline cells derived from MCF-7, MDAMB231 and T47D in the presence and absence of DOXY. The growth curves of MCF-7 and MDA-MB231 sublines were determined by MTT assay and the growth curves of T47D sublines were determined by cell counting. 


\section{Colony formation assay}
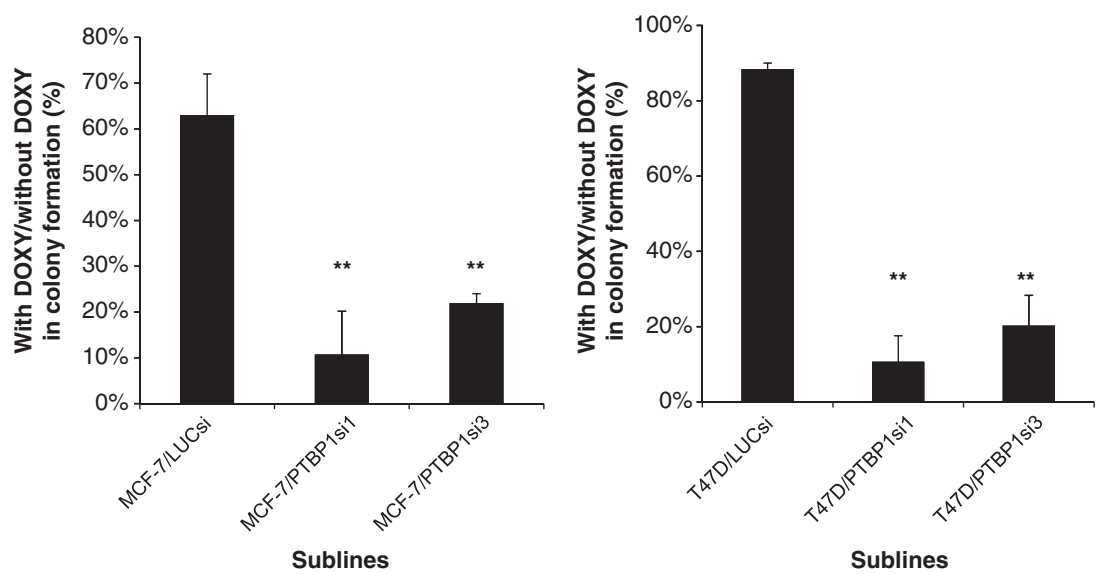

Figure 5. Knockdown of PTBP1 expression inhibits AIG. Average ratios (expressed in percentage) of colony numbers formed in the presence vs in the absence of DOXY $(n=4)$. Error bar: s.e.; ${ }^{* *} P<0.01$.

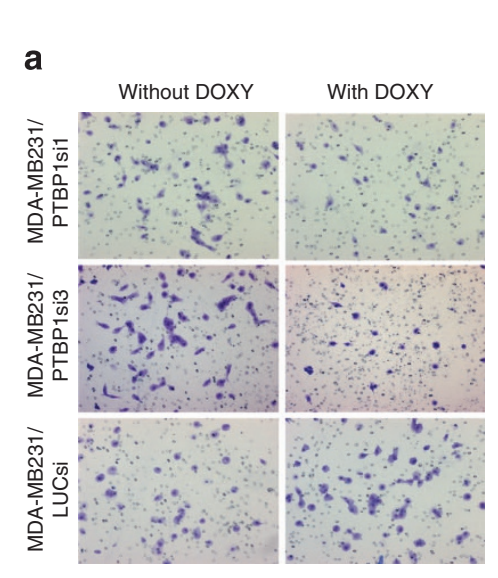

Invasion assay

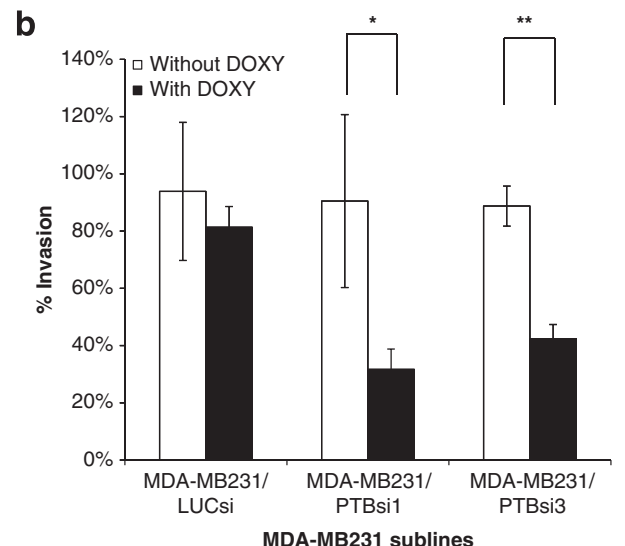

Figure 6. Knockdown of PTBP1 expression inhibits in vitro invasiveness of MDA-MB231 cells. (a) Representative pictures showing invasive cells under microscope $(\times 40)$; (b) Quantitation of cell invasiveness expressed as \% invasion (see the Materials and Methods for the definition of \% invasion; $n=3$ ). Error bar: s.e.; ${ }^{*} P<0.05 ;{ }^{*} P<0.01$.

four- to eightfold in PTBP1-knockdown cells compared with cells without PTBP1 knockdown. To determine whether the above change causes a shift of glucose metabolism toward oxidative phosphorylation, we measured oxygen consumptions in T47D sublines. As shown in Figure 7b, oxygen consumption is indeed increased in PTBP1-knockdown cells. These data suggest that PTBP1 knockdown-induced cell growth inhibition could be partially mediated by altered cellular metabolism.

\section{DISCUSSION}

We have extended our earlier studies on the role of PTBP1 in human ovarian cancer to its role in human breast cancer. We have shown herein that (i) PTBP1 is upregulated in the process of HMEC transformation; (ii) ectopic expression of PTBP1 alters HMEC morphology and enhances AIG; and (iii) overexpressed PTBP1 is required to maintain breast cancer cell growth and transformation properties such as AIG, aerobic glycolysis and invasiveness. Our present results indicate that PTBP1 likely has an important role in breast cancer as in ovarian cancer, suggesting that changes of PTBP1 expression, and the alternative splicing it regulates, may be not restricted to one or two tumor types but may be a universal phenomenon, at least for tumors of epithelial origin. The similarities between these two tumor types, especially triplenegative breast cancer and high-grade serous ovarian cancer, has been noted in the recent Cancer Genome Atlas (TCGA) study. ${ }^{35}$

Our results raise a question about why PTBP1 is associated with transformation and what mechanisms mediate its apparent role in tumorigenesis. Recent findings of alternative splicing of pyruvate kinase (PK) and how its splice variants control the metabolism of glucose in cancer cells shed some light on this matter. ${ }^{32}$ It has long been known that tumor cells metabolize glucose by aerobic glycolysis, whereas normal cells use oxidative phosphorylation (the Warburg effect). ${ }^{36}$ The difference is now attributed to the switch of the expression of the M1 isoform (PKM1) of PK to the expression of the M2 isoform (PKM2) in tumor cells, as the latter is necessary for aerobic glycolysis and promotes tumorigenesis, whereas the former functions in the opposite way, that is, enhancing oxidative phosphorylation and reducing tumorigenesis. $^{32}$ PKM1 and PKM2 are derived from a single PK gene through alternative splicing of two mutually exclusive exons (exon 9 and exon 10) with PKM1 (exon 9 included) normally expressed in differentiated cells and PKM2 (exon 10 included) mainly expressed in embryonic cells and cancer cells. ${ }^{32}$ PTBP1, together with the heterogenous nuclear ribonucleoproteins A1 and $\mathrm{A} 2$, promotes the expression of PKM2 by repressing the 

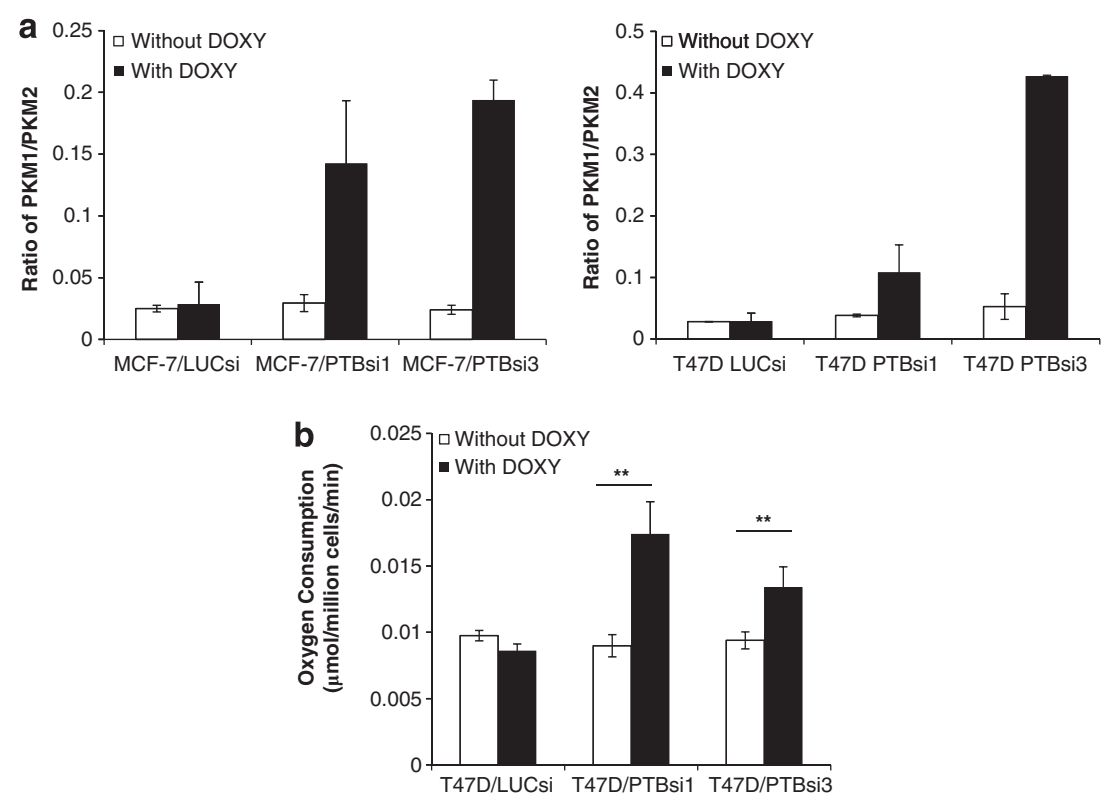

Figure 7. Knockdown of PTBP1 increases the expression of PKM1 but not PKM2. (a) Real-time PCR-determined relative quantitation of PKM1 and PKM2 mRNA levels in subline cells treated with or without DOXY. Shown are the average results and s.d. of two experiments. (b) Oxygen consumption assay of T47D sublines treated with or without DOXY. Shown are the results of three independent experiments. Error bar: s.e.; ${ }^{* *} P<0.01$

splicing of exon 9 and thus the expression of PKM1. ${ }^{33,34}$ Therefore, overexpression of PTBP1 is required for tumorigenesis at least partly because of its ability to promote aerobic glycolysis via upregulation of PKM2. Given that PTBP1 also regulates the expression and splicing of many other genes, ${ }^{37}$ it is very likely that there exist other mechanisms that mediate the actions of PTBP1 in cellular transformation.

As one example, the morphological changes (Figure 2c) of 184A1 cells after ectopic expression of the PTBP1-EGFP fusion protein is likely due to enhanced cell-cell adhesion. It was recently reported that depletion of PTBP1 caused loss of adherens junctions in the dorsal telencephalon, indicating PTBP1 has a role in the formation of these important cell junctions. ${ }^{38}$ Our results provide new evidence suggesting that PTBP1 is a regulator of cellcell adhesion. It remains to be determined what molecular pathways mediate this function of PTBP1 and how this function is related to PTBP1's capability to increase AIG of 184A1. We recently found that knockdown of PTBP1 in an ovarian cancer cell line A2780 caused downregulation of IQGAP1 (data not shown), a scaffolding protein that regulates multiple important cellular processes including cell-cell adhesion and cell migration. ${ }^{39}$ It was also reported previously that knockdown of IQGAP1 substantially inhibited AIG of MCF-7 cells. ${ }^{40}$ Therefore, it is possible that the effects of PTBP1 in this process are partly mediated by IQGAP1.

The observation that ectopic expression of PTBP1-EGFP did not promote $184 \mathrm{~A} 1$ cell growth (Figure $2 \mathrm{~b}$ ) appears contradictory to the growth inhibition of breast cancer cell lines after PTBP1 knockdown (Figure 4). However, this seeming discrepancy may well reflect the fact that the parental 184A1 cells (at passage 47) used in this study already achieved rapid growth ${ }^{41}$ and might have maximized their proliferative potential. Given the moderate upregulation of PTBP1 expression in the parental 184A1 compared with HMECs with finite lifespan (Figure 1), we speculate that high levels of PTBP1 are not necessary for maintenance of rapid cell growth but are critical for other transformation phenotypes such as AIG and invasiveness.

Finally, there is no evidence at present suggesting that PTBP1 is a driving force or an initiating factor of tumorigenesis. Our view is that PTBP1 may have intermediary role in the tumorigenic process by regulating the expression and/or splicing of other effector genes that directly give rise to the tumor cell phenotypes such as AIG, invasiveness and aerobic glycolysis. The upstream factors that control the expression of PTBP1 include c-Myc, N-Myc, E2f1, Nanog, Klf4 and microRNAs, ${ }^{42}$ all subjects of current investigation.

\section{MATERIALS AND METHODS}

\section{Cell culture}

The human breast cancer cell lines MCF-7, T47D and their sublines were grown in Dulbecco's modified Eagle's medium (DMEM) supplemented with $10 \%$ fetal bovine serum (FBS) and $2 \mathrm{~mm}$ L-glutamine at $37{ }^{\circ} \mathrm{C}, 5 \% \mathrm{CO}_{2}$. MDAMB231 and its sublines were grown in minimum essential medium supplemented with $10 \% \mathrm{FBS}$ and $2 \mathrm{~mm}$ L-glutamine at $37{ }^{\circ} \mathrm{C}, 5 \% \mathrm{CO}_{2}$. Finite and immortalized HMECs were grown in Clonetics MEBM (Lonza, Wilkersville, MD, USA) supplemented with $5 \mu \mathrm{g}$ insulin per $\mathrm{ml}, 70 \mu \mathrm{g}$ bovine pituitary extract per $\mathrm{ml}, 5 \mu \mathrm{g}$ transferrin per $\mathrm{ml}, 0.5 \mu \mathrm{g}$ hydrocortisone per $\mathrm{ml}, 5 \mathrm{ng}$ epidermal growth factor per $\mathrm{ml}$ and $10^{-5} \mathrm{M}$ isoproternol at $37^{\circ} \mathrm{C}, 0.2 \% \mathrm{CO}_{2}{ }^{25,43}$

\section{Western blotting}

Whole-cell lysates were prepared by adding T-PER Tissue Protein Extraction Reagent (Pierce Biotechnology, Rockford, IL, USA) supplemented with protease inhibitor cocktail ( $2 \mathrm{~mm}$ AEBSF, $1 \mathrm{~mm}$ EDTA, $130 \mu \mathrm{m}$ Bestatin, $14 \mu \mathrm{m}$ E-64, $1 \mathrm{~mm}$ Leupeptin and $0.3 \mu \mathrm{m}$ Aprotinin) (Sigma, St Louis, MO, USA) or by adding $1 \times$ sample buffer $(50 \mathrm{~mm}$ Tris $\mathrm{pH} 6.8,2 \%$ SDS, $10 \%$ glycerol, $5 \%$ $\beta$-mercaptoethanol and $0.002 \%$ bromophenol blue) to cells in petri dishes and washed once with $1 \times$ phosphate-buffered saline. The lysates made with T-PER Tissue Protein Extraction Reagent were centrifuged at $10000 \mathrm{~g}$ for $5 \mathrm{~min}$ to remove the cell debris, and the supernatants were collected and quantified for protein concentration by Bio-Rad Protein Assay reagent (Bio-Rad, Hercules, CA, USA). Western blotting was performed as described previously. 22

Establishment of stable cell lines expressing DOXY-induced PTBP1 siRNA

Lentiviral vectors were employed to introduce coding sequences for PTBP1 shRNA into the cells as described in our previous study. ${ }^{22}$ Two effective PTBP1 siRNAs, PTBP1siRNA1 and PTBP1siRNA3, were used in that study and their sequences were described in He et al. ${ }^{22}$ The control siRNA (LUCsiRNA) 
targets the luciferase gene, whose sequence is $5^{\prime}$-CTTACGCTGAGTACTTC $\mathrm{GA}-3^{\prime} .^{28}$ The establishment in the present study of stable breast cancer cell lines (called sublines) expressing DOXY-inducible PTBP1 siRNA was accomplished in two steps. First, parental cells (MCF-7, T47D and MDA-MB231) were transduced by lentiviruses carrying an expression cassette containing the regulatory protein TTR/KRAB and the reporter gene $d s R e d 2$. The positive clones expressing $d s R e d 2$ were then reinfected with lentiviruses carrying coding sequences for PTBP1 shRNA1, PTBP1shRNA3 or luciferase shRNA. The isolated cell colonies were picked and transferred to 24-well plates and grown in the presence or absence of DOXY $(1 \mu \mathrm{g} / \mathrm{ml})$. Positive cells were identified by the expression of both red fluorescent protein and GFP when grown with DOXY. The regulation by DOXY of siRNA expression in these infected cells was verified by measuring PTBP1 expression by western blotting.

\section{Real-time PCR}

Total RNAs were extracted with Trizol reagent (Invitrogen, Carlsbad, CA, USA) from sublines treated with or without DOXY for 5 days. Complementary DNA was synthesized from $2 \mu \mathrm{g}$ of total RNA with High Capacity cDNA Reverse Transcription Kit (Applied Biosystems/Invitrogen, Foster City, CA, USA). Real-time PCR was set up with Fast SYBR Green Master Mix (Applied Biosystems, Foster City, CA, USA) and run in StepOne Plus Real-Time PCR System (Applied Biosystems). The primer pairs for amplification of PK isoforms are the following:

M1 isoform: $5^{\prime}$-ACTATCCTCTGGAGGCTGTGCGC- $3^{\prime}$ and $5^{\prime}$ CGATTATGGCC CCACTGCAGCA-3'.

M2 isoform: $5^{\prime}$-TCTGGAGGCTGTGCGCATGC- $3^{\prime}$ and $5^{\prime}$-AAGCCTCCACGCT GCCCATG-3'.

The expression levels of PK isoforms were determined using the comparative CT $(\Delta \Delta C T)$ method ${ }^{44}$ with glyceraldehyde 3-phosphate dehydrogenase as the endogenous control, the sublines carrying the luciferase shRNA expression cassette without DOXY treatment served as references.

\section{Cloning of PTBP1-EGFP fusion protein}

The coding sequence for PTBP1 isoform was amplified from pcDNA3PTBP1(L) (He et al., unpublished data) with forward primer 5'-AGATCTATG GACGGCATTGTCCCAGA-3' and reverse primer $5^{\prime}$-CCGCGGGATGGTGGACTT GGAGAAGG-3'. The PCR product was first cloned into pCR-Blunt II-TOPO vector (Invitrogen) and then subcloned into pEGFP-N1 vector (Clontech, Mountain View, CA, USA) between Bglll and Sacll sites to form the coding sequence for PTBP1-EGFP fusion protein. Subsequently, the PTBP1-EGFPcoding sequence was subcloned into a lentiviral vector LV-THM ${ }^{27}$ between Pmel and Spel to replace the GFP-coding sequence in the vector, and the resulting plasmid was named LV-PTBP1-EGFP. Lentiviruses carrying the PTBP1-EGFP expression cassette was prepared as described previously. ${ }^{22}$

\section{Cell growth assay}

Cell growth was determined by a cell growth curve. One thousand cells per well were seeded in triplicate in 96-well plates and grown in media supplemented with or without DOXY $(1 \mu \mathrm{g} / \mathrm{ml})$ at $37{ }^{\circ} \mathrm{C}, 5 \% \mathrm{CO}_{2}$. One, two, five and six days after seeding, 3-(4,5-dimethylthiazol-2-yl)-2,5-diphenyltetrazolium bromide (MTT) assays were performed as described previously. ${ }^{22}$ For the T47D sublines, 4000 cells per well were seeded in duplicate in 24-well plates and the cell growth curve was determined by daily cell counting for 6 days using Multisizer Coulter Counter (Beckman Coulter, Inc., Brea, CA, USA).

\section{Colony formation on soft agar}

For breast cancer sublines, the assay was performed in six-well plates containing two layers of soft agar. The bottom layer was $0.4 \%$ agarose in DMEM supplemented with $5 \%$ FBS and the top layer was $0.3 \%$ agarose mixed with 2000 cells in DMEM supplemented with 5\% FBS and with or without DOXY $(1 \mu \mathrm{g} / \mathrm{ml})$. Once the soft agar was solidified, $1 \mathrm{ml}$ of DMEM supplemented with $5 \%$ FBS and with or without DOXY $(1 \mu \mathrm{g} / \mathrm{ml})$ was added to each well and the plates were left at $37^{\circ} \mathrm{C}, 5 \% \mathrm{CO}_{2}$ for $3-4$ weeks. For each subline, the assay was done in duplicate. Colonies on the soft agar were counted manually. For immortalized HMECs, the assay was performed in 96-well plates using CytoSelect 96-Well Cell Transformation Assay kit (Cell Biolabs, Inc., San Diego, CA, USA), according to the manufacturer's instructions. MCDB $170(2 \times$; United States Biological, Swampscott, MA, USA) supplemented with insulin $(10 \mu \mathrm{g} / \mathrm{ml})$, bovine pituitary extract $(140 \mu \mathrm{g} / \mathrm{ml})$, transferrin $(10 \mu \mathrm{g} / \mathrm{ml})$, hydrocortisone $(1 \mu \mathrm{g} / \mathrm{ml})$, human epidermal growth factor $(10 \mathrm{ng} / \mathrm{ml})$ and $2 \times 10^{-5} \mathrm{M}$ isoproternol were used to prepare the agar matrix in the assay.

\section{Cell invasion assay}

This assay was performed using BD BioCoat Matrigel Invasion Chamber (BD Biosciences, Bedford, MA, USA) according to the manufacturer's instructions. Briefly, $2.5 \times 10^{4}$ cells in $0.5 \mathrm{ml}$ of minimum essential medium without FBS were seeded into the rehydrated Matrigel inserts or control inserts, all of which were placed in the 24-well plates containing $0.5 \mathrm{ml}$ of minimum essential medium supplemented with $10 \% \mathrm{FBS}$. After incubation at $37{ }^{\circ} \mathrm{C}, 5 \% \mathrm{CO}_{2}$ for $22 \mathrm{~h}$, noninvading cells were removed from the inserts using cotton-tipped swabs. The invading cells on the lower surface of the inserts were then fixed in 100\% methanol and stained in $1 \%$ toluidine blue. Finally, the membranes were cut from the inserts and mounted on the microscope slides. Cells in five fields were counted manually at $\times 150$ magnification. The invasiveness of the cells was expressed as \% invasion, which equals the number of cells invading through the Matrigel membrane divided by the number of cells migrating through the control membrane.

\section{Oxygen consumption assay}

T47D subline cells were seeded at $10^{6}$ cells per $10-\mathrm{cm}$ dish in triplicate and grown in low-glucose (1g/l) DMEM supplemented with or without $10 \mathrm{ng} /$ $\mathrm{ml}$ of DOXY at $37^{\circ} \mathrm{C}, 5 \% \mathrm{CO}_{2}$ for 5 days. Cells were then washed with phosphate-buffered saline, collected and resuspended in $380 \mu \mathrm{l}$ of fresh DMEM. The rate of oxygen consumption was measured at $37^{\circ} \mathrm{C}$ using a Strathkelvin Model 782 oxygen meter (Strathkelvin Instruments Limited, North Lanarkshire, Scotland) equipped with a Clark-type oxygen electrode. Results are expressed as micromoles of oxygen consumed per minute per million cells. The cell number was determined using a Multisizer 3 Coulter Counter (Beckman Coulter, Inc., Indianapolis, IN, USA).

\section{CONFLICT OF INTEREST}

The authors declare no conflict of interest.

\section{ACKNOWLEDGEMENTS}

We thank Dr Didier Trono (University of Geneva, Switzerland) for his generous gift of lentiviral vectors LV-THM and LV-tTR/KRAB-Red as well as plasmids PMD2.G, pMDLg/ pRRE and pRSV-Rev; Dr Nissim Hay for the use of the Strathkelvin Model 782 oxygen meter; Dr Veronique Nogueira for technical assistance with the oxygen consumption assay. We also thank our colleague, Ms Martina Vaskova, for her outstanding administrative assistance. This work was supported in part by National Cancer Institute grants RO1 CA40570 and RO1 CA138762 to WTB and in part by the State of Illinois Department of Public Health's Penny Severns Breast, Cervical and Ovarian Cancer Research Fund to $\mathrm{XH}$. It was conducted in a facility constructed with support from the NCRR NIH Grant C06RR15482. MRS was supported by Department of Defense Grant BCRP BC060444 carried out at Lawrence Berkeley National Laboratory under Contract Number DEAC02-05CH1123.

\section{REFERENCES}

1 Siegel R, Naishadham D, Jemal A. Cancer statistics 2013. CA Cancer J Clin 2013; 63: 11-30.

2 Minn AJ, Gupta GP, Siegel PM, Bos PD, Shu W, Giri DD et al. Genes that mediate breast cancer metastasis to lung. Nature 2005; 436: 518-524.

3 Zuo T, Wang L, Morrison C, Chang X, Zhang H, Li W et al. FOXP3 is an X-linked breast cancer suppressor gene and an important repressor of the HER-2/ErbB2 oncogene. Cell 2007; 129: 1275-1286.

4 Boehm JS, Zhao JJ, Yao J, Kim SY, Firestein R, Dunn IF et al. Integrative genomic approaches identify IKBKE as a breast cancer oncogene. Cell 2007; 129: 1065-1079.

5 Han HJ, Russo J, Kohwi Y, Kohwi-Shigematsu T. SATB1 reprogrammes gene expression to promote breast tumour growth and metastasis. Nature 2008; 452 : 187-193.

6 Harris TJ, McCormick F. The molecular pathology of cancer. Nat Rev Clin Oncol 2010; 7: 251-265.

7 Tavazoie SF, Alarcon C, Oskarsson T, Padua D, Wang Q, Bos PD et al. Endogenous human microRNAs that suppress breast cancer metastasis. Nature 2008; 451: 147-152. 
8 Huang Q, Gumireddy K, Schrier M, le Sage C, Nagel R, Nair S et al. The microRNAs miR-373 and miR-520c promote tumour invasion and metastasis. Nat Cell Biol 2008; 10: 202-210.

9 Spellman R, Smith CW. Novel modes of splicing repression by PTB. Trends Biochem Sci 2006; 31: 73-76.

10 Castelo-Branco P, Furger A, Wollerton M, Smith C, Moreira A, Proudfoot N. Polypyrimidine tract binding protein modulates efficiency of polyadenylation. Mol Cell Biol 2004; 24: 4174-4183.

11 Hall-Pogar T, Liang S, Hague LK, Lutz CS. Specific trans-acting proteins interact with auxiliary RNA polyadenylation elements in the COX-2 3'-UTR. RNA 2007; 13 1103-1115.

12 Tillmar L, Carlsson C, Welsh N. Control of insulin mRNA stability in rat pancreatic islets. Regulatory role of a $3^{\prime}$-untranslated region pyrimidine-rich sequence. J Biol Chem 2002; 277: 1099-1106.

13 Tillmar L, Welsh N. Hypoxia may increase rat insulin mRNA levels by promoting binding of the polypyrimidine tract-binding protein (PTB) to the pyrimidine-rich insulin mRNA 3'-untranslated region. Mol Med 2002; 8: 263-272.

14 Coles LS, Bartley MA, Bert A, Hunter J, Polyak S, Diamond P et al. A multi-protein complex containing cold shock domain (Y-box) and polypyrimidine tract binding proteins forms on the vascular endothelial growth factor mRNA. Potential role in mRNA stabilization. Eur J Biochem 2004; 271: 648-660.

15 Hamilton BJ, Genin A, Cron RQ, Rigby WF. Delineation of a novel pathway that regulates CD154 (CD40 ligand) expression. Mol Cell Biol 2003; 23: 510-525.

16 Cote CA, Gautreau D, Denegre JM, Kress TL, Terry NA, Mowry KL. A Xenopus protein related to hnRNP I has a role in cytoplasmic RNA localization. Mol Cell 1999; 4: 431-437.

17 Auweter SD, Allain FH. Structure-function relationships of the polypyrimidine tract binding protein. Cell Mol Life Sci 2008; 65: 516-527.

18 Mitchell SA, Spriggs KA, Coldwell MJ, Jackson RJ, Willis AE. The Apaf-1 internal ribosome entry segment attains the correct structural conformation for function via interactions with PTB and unr. Mol Cell 2003; 11: 757-771.

19 Song Y, Tzima E, Ochs K, Bassili G, Trusheim H, Linder M et al. Evidence for an RNA chaperone function of polypyrimidine tract-binding protein in picornavirus translation. RNA 2005; 11: 1809-1824.

20 Patton JG, Mayer SA, Tempst P, Nadal-Ginard B. Characterization and molecular cloning of polypyrimidine tract-binding protein: a component of a complex necessary for pre-mRNA splicing. Genes Dev 1991; 5: 1237-1251.

$21 \mathrm{He} \mathrm{X}$, Ee PL, Coon JS, Beck WT. Alternative splicing of the multidrug resistance protein 1/ATP binding cassette transporter subfamily gene in ovarian cancer creates functional splice variants and is associated with increased expression of the splicing factors PTB and SRp20. Clin Cancer Res 2004; 10 4652-4660.

22 He X, Pool M, Darcy KM, Lim SB, Auersperg N, Coon JS et al. Knockdown of polypyrimidine tract-binding protein suppresses ovarian tumor cell growth and invasiveness in vitro. Oncogene 2007; 26: 4961-4968.

23 Jin W, McCutcheon IE, Fuller GN, Huang ES, Cote GJ. Fibroblast growth factor receptor-1 alpha-exon exclusion and polypyrimidine tract-binding protein in glioblastoma multiforme tumors. Cancer Res 2000; 60: 1221-1224.

24 Wang C, Norton JT, Ghosh S, Kim J, Fushimi K, Wu JY et al. Polypyrimidine tractbinding protein (PTB) differentially affects malignancy in a cell line-dependent manner. J Biol Chem 2008; 283: 20277-20287.
25 Stampfer MR, Bartley JC. Induction of transformation and continuous cell lines from normal human mammary epithelial cells after exposure to benzo[a]pyrene. Proc Natl Acad Sci USA 1985; 82: 2394-2398.

26 Stampfer MR, Garbe J, Nijjar T, Wigington D, Swisshelm K, Yaswen P. Loss of p53 function accelerates acquisition of telomerase activity in indefinite lifespan human mammary epithelial cell lines. Oncogene 2003; 22: 5238-5251.

27 Wiznerowicz M, Trono D. Conditional suppression of cellular genes: lentivirus vector-mediated drug-inducible RNA interference. J Virol 2003; 77: 8957-8961.

28 Elbashir SM, Harborth J, Lendeckel W, Yalcin A, Weber K, Tuschl T. Duplexes of 21nucleotide RNAs mediate RNA interference in cultured mammalian cells. Nature 2001; 411: 494-498.

29 Hanahan D, Weinberg RA. Hallmarks of cancer: the next generation. Cell 2011; 144: 646-674.

30 Pishvaian MJ, Feltes CM, Thompson P, Bussemakers MJ, Schalken JA, Byers SW. Cadherin-11 is expressed in invasive breast cancer cell lines. Cancer Res 1999; 59: 947-952.

31 Koppenol WH, Bounds PL, Dang CV. Otto Warburg's contributions to current concepts of cancer metabolism. Nat Rev Cancer 2011; 11: 325-337.

32 Christofk HR, Vander Heiden MG, Harris MH, Ramanathan A, Gerszten RE, Wei R et al. The M2 splice isoform of pyruvate kinase is important for cancer metabolism and tumour growth. Nature 2008; 452: 230-233.

33 Clower CV, Chatterjee D, Wang Z, Cantley LC, Vander Heiden MG, Krainer AR. The alternative splicing repressors hnRNP A1/A2 and PTB influence pyruvate kinase isoform expression and cell metabolism. Proc Natl Acad Sci USA 2010; 107: 1894-1899.

34 David CJ, Chen M, Assanah M, Canoll P, Manley JL. HnRNP proteins controlled by c-Myc deregulate pyruvate kinase mRNA splicing in cancer. Nature 2010; 463 364-368.

35 Cancer Genome Atlas Network. Comprehensive molecular portraits of human breast tumours. Nature 2012; 490: 61-70.

36 Warburg O. On the origin of cancer cells. Science 1956; 123: 309-314.

37 Arslan AD, He X, Wang M, Rumschlag-Booms E, Rong L, Beck WT. A highthroughput assay to identify small-molecule modulators of alternative pre-mRNA splicing. J Biomol Screening 2013; 18: 180-190.

38 Shibasaki T, Tokunaga A, Sakamoto R, Sagara H, Noguchi S, Sasaoka T et al. PTB deficiency causes the loss of adherens junctions in the dorsal telencephalon and leads to lethal hydrocephalus. Cereb Cortex 2012; 23: 1824-1835.

39 Noritake J, Watanabe T, Sato K, Wang S, Kaibuchi K. IQGAP1: a key regulator of adhesion and migration. J Cell Sci 2005; 118: 2085-2092.

40 Jadeski L, Mataraza JM, Jeong HW, Li Z, Sacks DB. IQGAP1 stimulates proliferation and enhances tumorigenesis of human breast epithelial cells. J Biol Chem 2008; 283: 1008-1017.

41 Stampfer MR, Bodnar A, Garbe J, Wong M, Pan A, Villeponteau B et al. Gradual phenotypic conversion associated with immortalization of cultured human mammary epithelial cells. Mol Biol Cell 1997; 8: 2391-2405.

42 Chen M, Zhang J, Manley JL. Turning on a fuel switch of cancer: hnRNP proteins regulate alternative splicing of pyruvate kinase mRNA. Cancer Res 2010; 70: 8977-8980.

43 Hammond SL, Ham RG, Stampfer MR. Serum-free growth of human mammary epithelial cells: rapid clonal growth in defined medium and extended serial passage with pituitary extract. Proc Natl Acad Sci USA 1984; 81: 5435-5439.

44 Schmittgen TD, Livak KJ. Analyzing real-time PCR data by the comparative C(T) method. Nat Protoc 2008; 3: 1101-1108. 